\title{
INDEX TO VOLUME 1
}

\section{RESEARCH-EXPOSITORY PAPERS}

Aschbacher, Michael, An introduction to the classification of finite simple groups, 39

Auslander, L. and Tolimieri, R., Is computing with the finite Fourier transform pure or applied mathematics, 847

Brauer, Richard, Blocks of characters and structure of finite groups, 21

Brauer, Richard D. See Feit, Walter

Cohen, Joel E., Ergodic theorems in demography, 275

Curtis, Charles W., Representations of finite groups of Lie type, 721

Ekeland, Ivar, Nonconvex minimization problems, 443

Feit, Walter, Richard D. Brauer, 1

Fowler, D. H., Ratio in early Greek mathematics, 807

Gorenstein, Daniel. The classification of finite simple groups I. Simple groups and local analysis, 43

Griffiths, Phillip A., Complex analysis and algebraic geometry, 595

Knuth, Donald E., Mathematical typography, 337

Orlik, Peter, Singularities and group actions, 703

Stanley, Richard P., Invariants of finite groups and their applications to combinatorics, 475

Tolimieri, R. See Auslander, L.

Wells, R. O., Jr., Complex manifolds and mathematical physics, 296

\section{RESEARCH ANNOUNCEMENTS}

Agler, Jim, An invariant subspace theorem, 425

Alvis, Dean, The duality operation in the character ring of a finite Chevalley group, 907

Amitsur, S. A., Rowen, L. H. and Tignol, J. P., Division algebras of degree 4 and 8, 691

Berger, M. S. and Church, P. T., On the complete integrability of nonlinear partial differential equations, 698

Bichteler, Klaus, Stochastic integrators, 761

Block, Richard E., Classification of the irreducible representations of $81(2, \mathrm{C}), 247$

Block, Richard E. and Wilson, Robert Lee, The restricted simple Lie algebras with a two-dimensional Cartan subalgebra, 682

Cappell, Sylvain E. and Shaneson, Julius L., Linear algebra and topology, 685

Cappell, Sylvain E. and Shaneson, Julius L., Nonlinear similarity of matrices, 899

do Carmo, M. and Peng, C. K., Stable complete minimal surfaces in $R^{3}$ are planes, 903

César de Sá, Eugénia and Rourke, Colin, The homotopy type of homeomorphisms groups of 3-manifolds, 251

Chacon, R. V. and Jamison, Benton, Traversal times of Markov processes, 583

Charney, Ruth M., Homology stability of $G L_{n}$ of a Dedekind domain, 428

Church, P. T. See Berger, M. S.

Connes, Alain and Moscovici, Henri, The $L^{2}$-index theorem for homogeneous spaces, 688

Córdoba, Antonio, Maximal functions: A proof of a conjecture of A. Zygmund, 255

Fein, Burton, Schacher, Murray and Sonn, Jack, Brauer groups of rational function fields, 766

Ferguson, H. R. P. and Forcade, R. W., Generalization of the Euclidean algorithm for real num. bers to all dimensions higher than two, 912

Forcade, R. W. See Ferguson, H. R. P.

Fritsch, Rudolf and Latch, Dana May, Homotopy inverses for nerve, 258

Grunewald, Fritz J. and Segal, Daniel, The solubility of certain decision problems in arithmetic and algebra, 915

Grunewald, F. J., Pickel, P. F. and Segal, D., Finiteness theorems for polycyclic groups, $\mathbf{5 7 5}$

Hardt, Robert and Simon, Leon, Boundary regularity and embedded solutions for the oriented plateau problem, 263

Heath-Brown, D. R. and Iwaniec, H., On the difference between consecutive primes, 758

Iwaniec, H. See Heath-Brown, G. R.

Jamison, Benton. See Chacon, R. V. 
Jørgensen, Palle E. T., Selfadjoint operator extensions satisfying the Weyl commutation relations, 266

Kailath, T., Kung, S-Y. and Morf, M., Displacement ranks of a matrix, 769

Keane, M. and Smorodinsky, M., The finitary isomorphism theorem for Markov shifts, 436

Kenig, Carlos E. and Tomas, Peter A., On conjectures of Rivière and Strichartz, 694

Kung, S-Y. See Kailath, T.

Lakshmibai, V.,Musili, G. and Seshadri, C. S., Geometry of G/P, 432

Latch, Dana May. See Fritsch, Rudolf

Morf, M., Kailath, T. See Kung, S-Y

Moscovici, Henri. See Connes, Alain

Musili, C. and Lakshmibai, V. See Seshadri, C. S.

Peng, C. K. See do Carmo, M.

Pickel, P. F. See Grunewald, F. J.

Quinn, Frank, Ends of maps and applications, 270

Rourke, Colin. See César de Sá, Eugénia

Rowen, L. H. See Amitsur, S. A.

Schacher, Murray. See Fein, Burton

Segal, D. See Grunewald, F. J.

Segal, Daniel. See Grunewald, Fritz J.

Seshadri, C. S. See Lakshmibai, V.

Shaneson, Julius L. See Cappell, Sylvain E.

Shaneson, Julius L. See Cappell, Sylvain E.

Simon, Leon. See Hardt, Robert

Smorodinsky, M. See Keane, M.

Sonn, Jack. See Fein, Burton

Swarup, G. A., Two reductions of the Poincaré conjecture, 774

Tenenblat, Keti and Terng, Chuu-Lian, A higher dimension generalization of the Sine-Gordon equation and its Bäcklund transformation, 589

Terng, Chuu-Lian. See Tenenblat, Keti

Tignol, J. P. See Amitsur, S. A.

Tomas, Peter A. See Kenig, Carlos E.

Uhlenbeck, Karen Keskulla, Removable singularities in Yang-Mills fields, 579

Vigfússon, Gudmundur, The queer differential equations for adiabatic compression of plasma, 778

Wagreich, Philip, Automorphic forms and singularities of complex surfaces, 585

Wilson, Robert E. See Block, Richard E.

\section{BOOK REVIEWS}

Adams, J. F. See May, J. P.

Ahern, Patrick, Abstract analytic function theory and Hardy algebras by Klaus Barbey and Heinz König, 201

Akin, E. See Graff, Richard

Aliprantis, Charalambos D. See Bernau, Simon J.

Andrews, George E., The collected papers of Alfred Young, 1873-1940, G. de B. Robinson (editor), 989

Andrews, George E. See Askey, Richard

Apostol, Tom M. See Knopp, Marvin, I.

Arsenin, Y. See Levine, Howard A.

Askey, Richard, The theory of partitions by George E. Andrews, 203

Babbitt, D. G., Thermodynamic formalism: The mathematical structures of classical equilibrium statistical mechanics, by David Ruelle, 931

Bănică, Constantin. See Hartshorne, Robin

Barbey, Klaus. See Ahern, Patrick

Barndorff-Nielsen, O. See Pitcher, T. S.

Barnhill, Robert E., Finite element Galerkin methods for differential equations by Graeme Fairweather, 245

Baues, Hans J. See Hilton, Peter J.

Baumgartner, James E., Combinatorial set theory by Neil H. Williams, 217

Baumslag, Gilbert, Combinatorial group theory by R. C. Lyndon and P. E. Schupp, 949 
Bear, H. S., Introduction to operator theory. I. Elements of functional analysis by Arlen Brown and Carl Pearcy, 220

Beckenstein, E. See Williamson, J. H.

Bell, J. L. See Eklof, Paul C.

Berberian, S. K., Topological uniform structures by Warren Page, 974

Berger, Melvin S. See Fitzpatrick, P. M.

Bergman, George, Skew field constructions by P. M. Cohn, 414

Berkson, Earl R., Spectral theory of linear operators by H. R. Dowson, 795

Bernau, Simon J., Locally solid Riesz spaces by Charalambos D. Aliprantis and Owen Burkinshaw, 997

Bigard, Alain. See Fuchs, Laszlo

Birch, B. J. See Grosswald, Emil

Birkhoff, Garrett, General lattice theory by George Grätzer. 789

Boas, R. P., Bessel polynomials by Emil Grosswald, 799

Bochner, Salomon, Gesammelte schriften by Gustav Herglotz, 1014

Boltianskǐ, Vladimir G. See Honsberger, Ross A.

Brezinski, C. See Henrici, Peter

Brown, Arlen. See Bear, H. S.

Brown, Harold. See Coxeter, H.S. M.

Brualdi, Richard A., Permanents by Henryk Minc, 965

Bülow, Rolf. See Coxeter, H.S. M.

Burkinshaw, Owen. See Bernau, Simon J.

Calabi, Eugenio, The Minkowskii multidimensional problem by Aleksey Vasil'yevich

Pogorelov, 636

Chow, Yutze. See Wonenburger, Maria

Ciarlet, Philippe G. See Fix, George J.

Clifford, A. H., Quasi-ideals in rings and semigroups by Ottó Steinfeld, 804

Cohn, P. M. See Bergman, George

Cooper, J. B. See Semadeni, Zbigniew

Coxeter, H.S. M., Crystallographic groups of four-dimensional space by Harold Brown, Rolf Bülow, Joachim Neubüser, Hans Wondratschek and Hans Zassenhaus, 792

Cristescu, Romulus. See Gretsky, Neil

Crossley, J. N., Mathematical logic. An introduction to model theory by A. H. Lightstone, 1003

Davis, M. H. A. See Fleming, Wendell H.

De Wilde, M. See Husain, Taqdir

Diestel, Joseph, The Bochner integral by Jan Mikusiński, 538

Dieudonné, Jean. See Halmos, P. R.

Dowson, H. R. See Berkson, Earl R.

Eklof, Paul C., Boolean-valued models and independence proofs in set theory by J. L. Bell, 221

Ewing, John H., Homology and cohomology theory by William S. Massey, 985

Faden, Arnold M. See Varian, Hal R.

Fairweather, Graeme. See Barnhill, Robert E.

Feferman, Solomon, Proof theory by Kurt Schütte, 244

Fell, J.M.G. See Rieffel, Marc A.

Fitzpatrick, P. M., Nonlinearity and functional analysis by Melvin S. Berger, 210

Fix, George J., The finite element method for elliptic problems by Philippe G. Ciarlet, 800

Flanders, Harley, Differential forms in mathematical physics by C. von Westenholz, 944

Fleming, Wendell H., Linear estimation and stochastic control by M.H. A. Davis, 228

Fritzsche, K. See Shiffman, Bernard

Fuchs, Laszlo, Groupes et anneaux réticulés by Alain Bigard, Klaus Keimel and Samuel Wolfenstein, 787

Goldstine, Herman H. See Parlett, B. N.

Golubitsky, Martin, Catastrophe theory and its applications by Tim Poston and Ian Stewart, 524

Good, I. J., Mechanizing hypothesis formation. Mathematical foundations for a general theory by P. Hájek and T. Havránek, 650

Goto, Morikuni. See Humphreys, J. E.

Graff, Richard, The metric theory of Banach manifolds, by E. Akin, 960

Grätzer, George. See Birkhoff, Garrett 
Grauert, H. See Shiffman, Bernard

Graver, J. E. See Mirsky, L.

Green, Leon, Lectures on closed geodesics by Wilhelm Klingenberg, 568

Gretsky, Neil, Ordered vector spaces and linear operators by Romulus Cristescu, 373

Grosshans, Frank D. See Humphreys, J. E.

Grosswald, Emil, The collected works of Harold Davenport edited by B.J. Birch, H. Halberstam and C. A. Rogers, 668

Grosswald, Emil. See Boas, R. P.

Guillemin, V. See Weinstein, Alan and Marsden, Jerrold E.

Gustafson, W. H., Topics in group rings by Sudarshan K. Sehgal, 654

Hájek, P. See Good, I. J.

Halberstam, H. See Grosswald, Emil

Halmos, P. R., Panorama des mathématiques pures. Le choix bourbachique, by Jean Dieudonné, 678

Halmos, P. R. See Zaanen, Adriaan

Hartshorne, Robin. See Shatz, Stephen S.

Hartshorne, Robin, Algebraic geometry I: Complex projective varieties by David Mumford, 513

Hartshorne, Robin, Algebraic methods in the global theory of complex spaces by Constantin Bănică and Octavian Stănăsilă, 566

Havránek, T. See Good, I. J.

Hay man, W. K. See Helms, L. L.

Helms, L. L., Subharmonic functions by W. K. Hayman and the late P. B. Kennedy, 376

Henrici, Peter, Accélération de la convergence en analyse numérique by $\mathrm{C}$. Brezinski, 639

Herb, Rebecca A., Harmonic analysis on real reductive groups by V. S. Varadarajan, 398

Herglotz, Gustav. See Bochner, Salomon

Hilton, Peter J., Obstruction theory on homotopy classification of maps by Hans J. Baues, 393

Honsberger, Ross A., Hilbert's third problem by Vladimir G. Boltiánskiī, 646

Howard, Ronald A., Fundamentals of decision analysis by Irving H. LaValle, 784

Humphreys, J. E., Semisimple Lie algebras by Morikuni Goto and Frank D. Grosshans, 515

Husain, Taqdir, Closed graph theorems and webbed spaces by M. De Wilde, 518

Israel, R. B. See Lenard, Andrew

James, D. G., Symplectic groups by O. T. O'Meara, 782

Johnson, W. B., Classical Banach spaces. I, by Joram Lindenstrauss and Lior Tzafriri, 230

Johnstone, P. T. See Mac Lane, Saunders

Karoubi, Max. See Stong, Robert E.

Keimel, Klaus. See Fuchs, Laszlo

Kennedy, P. B. See Helms, L. L.

Kleinberg, Eugene M. See Steel, John

Klingenberg, Wilhelm. See Green, Leon

Knopp, Marvin I., Modular forms and functions by Robert A. Rankin. Modular functions and Dirichlet series in number theory by Tom M. Apostol, 935

Kon, Masahiro. See Reilly, Robert C.

Konig, Heinz. See Ahern, Patrick

Koornwinder, Tom H., Symmetry and separation of variables by Willard Miller, Jr., 1020

Lallement, Gerard, Automata-theoretic aspects of formal power series by Arto Salomaa and Matti Soittola, 675

Lambek, Joachim, Introduction to categories, homological algebra and sheaf cohomology by Jan R. Strooker, 919

LaValle, Irving H. See Howard, Ronald A.

Lenard, Andrew, Convexity in the theory of lattice gases by R. B. Israel, 928

Levine, Howard A., Solutions of ill posed problems by N. Tikhonov and V. Y. Arsenin, 521

Lightstone, A. H. See Crossley, J. N.

Lindenstrauss, Joram. See Johnson, W. B.

Lloyd, N. G. See Nussbaum, Roger D.

Lyndon, R. C. See Baumslag, Gilbert

Mac Lane, Saunders, Topos theory by P. T. Johnstone, 1005

Manin, Yu. I. See Shoenfield, J. R.

Marsden, Jerrold E. and Weinstein, Alan, Geometric asymptotics by Victor Guillemin and 
Massey, William S. See Ewing, John H.

May, J. P., Infinite loop spaces by J. F. Adams, 42

Mikusiński, Jan. See Diestel, Joseph

Miller, Willard, Jr. See Koornwinder, Tom H.

Minc, Henryk. See Brualdi, Richard A.

Mirsky, L., Combinatorics with emphasis on the theory of graphs by J. E. Graver and M. E. Watkins, 380

Mumford, David. See Hartshorne, Robin

Nadler, Sam B., Jr. See Segal, Jack

Narici, L. See Williamson, J. H.

Neubüser, Joachim. See Coxeter, H.S. M.

Nussbaum, Roger D., Degree theory by N. G. Lloyd, 632

O'Meara, O. T. See James, D. G.

Orey, Steven, Innovation processes by Yury A. Rozanov, 532

Page, Warren. See Berberian, S. K.

Parlett, B. N., A history of numerical analysis from the 16 th through the 19 th century by $\mathrm{H}$. H. Goldstine, 388

Passman, Donald S. See Wallace, D. A. R.

Pearcy, Carl. See Bear, H. S.

Pitcher, T. S., Information and exponential families in statistical theory by O. Barndorff-Nielsen, 667

Pogorelov, Vasil'yevich, Aleksey. See Calabi, Eugenio

Poston, Tim. See Golubitsky, Martin

Prolla, Joao B. See Summers, W. H.

Protter, Murray H., Equations of mixed type by M. M. Smirnov, 534

Reilly, Robert C., Anti-invariant submanifolds by Kentaro Yano and Masahiro Kon, 627

Rieffel, Marc A., Induced representations and Banach*-algebraic bundles by J. M. G. Fell, 232

Rogers, C. A. See Grosswald, Emil

Rozanov, Yuriy A. See Orey, Steven

Ruelle, David. See Babbitt, D. G.

Salomaa, Arto. See Lallement, Gerard

Schechter, Martin. See Taylor, Michael E.

Schupp, P. E. See Baumslag, Gilbert

Schütte, Kurt. See Feferman, Solomon

Segal, Jack, Hyperspaces of sets by Sam B. Nadler, Jr., 412

Sehgal, Sudarshan K. See Gustafson, W. H.

Semadeni, Zbigniew, Saks spaces and applications to functional analysis by J. B. Cooper, 541

Shatz, Stephen S., Algebraic geometry by Robin Hartshorne, 553

Shiffman, Bernard, Several complex variables by H. Grauert and K. Fritzsche, 563

Shiryayev, A. N. See Siegmund, D. O.

Shoenfield, J. R., A course in mathematical logic by Yu. I. Manin, 539

Siegmund, D. O., Optimal stopping rules by A. N. Shiryayev, 570

Smirnov, M. M. See Protter, Murray H.

Soittola, Matti. See Lallement, Gerard

Stănăsilă, Octavian. See Hartshorne, Robin

Statman, Richard, Choice sequences. A chapter of intuitionistic mathematics by A. S. Troelstra

Steel, John, Infinitary combinatorics and the axiom of determinateness by Eugene M. Kleinberg, 560

Steinfeld, Otto. See Clifford, A. H.

Sternberg, S. See Weinstein, Alan and Marsden, Jerrold E.

Sternberg, Shlomo. See Guillemin, Victor

Stewart, Ian. See Martin, Golubitsky

Stong, Robert E., K-theory, an introduction by Max Karoubi, 658

Strooker, Jan R. See Lambek, Joachim

Suffel, C. See Williamson, J. H.

Summers, W. H., A pproximation of vector valued functions by Joao B. Prolla, 420

Sunder, V. S. See Zaanen, Adriaan

Taibleson, M. H., Non-Archimedean functional analysis by A.C.M. Van Rooij, 802 
Taylor, Michael E., Modern methods in partial differential equations, an introduction by Martin Schechter, 661

Tikhonov, N. See Levine, Howard A.

Troelstra, A. S. See Statman, Richard

Tzafriri, Lior. See Johnson, W. B.

Van Leunen, Marie-Claire. See Waterhouse, William C.

Van Rooij, A.C.M. See Taibleson, M. H.

Varadarajan, V.S. See Herb, Rebecca A.

Varian, Hal R., The economics of space and time by Arnold M. Faden, 390

Von Westenholz, C. See Flanders, Harley

Wallace, D. A. R., The algebraic structure of group rings by Donald S. Passman, 402

Wallach, Nolan R. See Marsden, Jerrold E.

Waterhouse, William C., $A$ handbook for scholars by Marie-Claire van Leunen, 411

Watkins, M. E. See Mirsky, L.

Weinstein, Alan. See Marsden, Jerrold E.

Williams, Neil H. See Baumgartner, James E.

Williamson, J. H., Topological algebras by E. Beckenstein, L. Narici and C. Suffel, 237

Wolfenstein, Samuel. See Fuchs, Laszlo

Wondratschek, Hans. See Coxeter, H.S. M.

Wonenburger, Maria, General theory of Lie algebras by Yutze Chow, 978

Yano, Kentaro. See Reilly, Robert C.

Zaanen, Adriaan, Bounded integral operators on $L^{2}$ spaces by P. R. Halmos and V. S. Sunder, 953

Zassenhaus, Hans. See Coxeter, H.S.M. 